\title{
Existence of positive periodic solutions for third-order differential equation with strong singularity
}

\section{Zhibo Cheng*}

"Correspondence:

czbo@hpu.edu.cn

School of Mathematics and

Information Science, Henan

Polytechnic University, Jiaozuo,

454003, China

\begin{abstract}
Sufficient conditions are presented for the existence of positive periodic solutions for a third-order nonlinear differential equation with singularity. Besides, an example is given to illustrate the results.

MSC: 34K13; 34B16; 34B18
\end{abstract}

Keywords: third order; singularity; positive periodic solution; topological degree theorem

\section{Introduction}

In this paper, we consider the following third-order differential equation with singularity:

$$
x^{\prime \prime \prime}+f\left(t, x^{\prime}\right) x^{\prime \prime}+h(t, x) x^{\prime}=g(t, x),
$$

where $f, h$ are continuous function and $T$-periodic about $t, h(t, x) \leq 0, g:[0, T] \times(0, \infty) \rightarrow$ $\mathbb{R}$ is an $L^{2}$-Carathéodory function, i.e., it is measurable in the first variable and continuous in the second variable, and for every $0<r<s$ there exists $h_{r, s} \in L^{2}[0, \omega]$ such that $|f(t, x(t))| \leq h_{r, s}$ for all $x \in[r, s]$ and a.e. $t \in[0, \omega], f$ is $\omega$-periodic function about $t$. Equation (1.1) is singular at 0 , which means that $g(t, x)$ becomes unbounded when $x \rightarrow 0^{+}$. We say that (1.1) is of repulsive type (resp. attractive type) if $g(t, x) \rightarrow+\infty$ (resp. $g(t, x) \rightarrow-\infty$ ) when $x \rightarrow 0$.

The study of singular differential equations began with the paper of Taliaferro. In 1979, Taliaferro [1] discussed the model equation with singularity

$$
y^{\prime \prime}+\frac{q(t)}{y^{\alpha}}=0, \quad 0<t<1
$$

subject to

$$
y(0)=0=y(1),
$$

and obtained the existence of a solution for the problem. Here $\alpha>0, q \in C(0,1)$ with $q>0$ on $(0,1)$ and $\int_{0}^{1} t(1-t) q(t) d t<\infty$. We call the equation a strong force condition if $\alpha \geq 1$ and we call it a weak force condition if $0<\alpha<1$.

@2014 Cheng; licensee Springer. This is an Open Access article distributed under the terms of the Creative Commons Attribution License (http://creativecommons.org/licenses/by/2.0), which permits unrestricted use, distribution, and reproduction in any medium, provided the original work is properly cited. 
Taliaferro's work has attracted the attention of many specialists in differential equations and they have contributed to the research of singular differential equations (see, e.g., [2-10]). Among these results, some are obtained for a second-order equation with strong force condition; see, e.g., [5, 9]. With a strong singularity, the energy near the origin becomes infinite and this fact is helpful for obtaining either a priori bounds, which are needed for a classical application of the degree theory, or the fast rotation, which is needed in recent versions of the Poincaré-Birkhoff theorem. Afterwards, in 2007 Torres [10] considered the periodic problem for a singular second-order equation with the weak force condition and showed that weak singularities may help periodic solutions to exist, which has driven the study of weak singularities (see [7]).

At the beginning, most of work concentrated on second-order singular differential equation, as in the references we mentioned above. Recently there have been published some results on third-order singular differential equation (see [11-17]). For example, in [13], Sun and Liu considered the singular nonlinear third-order periodic boundary value problem

$$
u^{\prime \prime \prime}+\rho^{3} u=f(t, u), \quad 0 \leq t \leq 2 \pi
$$

with $u^{(i)}(0)=u^{(i)}(2 \pi), i=0,1,2$, where $\rho \in(0,1 / \sqrt{3})$ and $f$ is singular at $t=0, t=1$, and $u=0$. Under suitable growth conditions, it is proved by constructing a special cone in $C[0,2 \pi]$ and employing fixed point index theory that the problem has at least one solution or at least two positive solutions. Afterwards, Li [15] investigated the third-order ordinary differential equation

$$
u^{\prime \prime \prime}(t)=f\left(t, u(t), u^{\prime}(t), u^{\prime \prime}(t)\right), \quad t \in \mathbb{R},
$$

where $f \in C(\mathbb{R} \times(0, \infty) \times \mathbb{R} \times \mathbb{R})$ is $\omega$-periodic in $t$, and $f(t, u, v, w)$ may be singular at $u=0$. By applying of a fixed point theorem in cones, the author obtained that existence results of positive $\omega$-periodic solutions for (1.4). Recently, Ren et al. [17] studied the third-order nonlinear singular differential equation

$$
x^{\prime \prime \prime}(t)+a x^{\prime \prime}(t)+b x^{\prime}(t)+c x(t)=f(t, x(t))+e(t) .
$$

Using Green's function for a third-order differential equation and some fixed point theorems, i.e., the Leray-Schauder alternative principle and Schauder's fixed point theorem, they established three new existence results of periodic solutions for (1.5).

Based on the above work, in this paper we will study (1.1) and obtain the existence of periodic solutions by using topological degree theorem. The rest of this paper is organized as follows. In Section 2, we give some lemmas. In Section 3, by using topological degree theorem by Mawhin [18], some sufficient conditions are obtained for the existence of positive periodic solutions of (1.1). We, respectively, consider repulsive type and attractive type. In Section 4, an example is given to show the feasibility of the main result of this paper.

\section{Some lemmas}

Lemma 2.1 [18, Theorem 2.4] Let $X, Y$ be real normed spaces and $L: D(L) \subset X \rightarrow Y$ a linear Fredholm map of index zero. Assume that $\Omega \subset X$ is an open bounded set and 
$N: \bar{\Omega} \rightarrow Y$ is an L-compact mapping. Assume that the following conditions are satisfied:

(i) $L x+\lambda N x \neq 0$, for each $(x, \lambda) \in[(D(L) \backslash \operatorname{ker} L) \cap \partial \Omega] \times(0,1)$;

(ii) $N x \notin \operatorname{Im} L$, for each $x \in \operatorname{ker} L \cap \partial \Omega$;

(iii) $D_{0}\left(\left.Q N\right|_{\operatorname{ker} L}, \Omega \cap \operatorname{ker} L\right) \neq 0$, where $Q: Y \rightarrow Y$ is a continuous projector such that $\operatorname{ker} Q=\operatorname{Im} L$ and $D_{0}$ is the Brouwer degree,

then the equation $L x+N x=0$ has at least one solution in $D(L) \cap \bar{\Omega}$.

For the sake of convenience, throughout this paper we will adopt the following notation:

$$
\begin{aligned}
& |u|_{\infty}=\max _{t \in[0, T]}|u(t)|, \quad|u|_{0}=\min _{t \in[0, T]}|u(t)|, \\
& |u|_{p}=\left(\int_{0}^{T}|u|^{p} d t\right)^{\frac{1}{p}}, \quad \bar{h}=\frac{1}{T} \int_{0}^{T} h(t) d t .
\end{aligned}
$$

Lemma 2.2 [19] If $\omega \in C^{1}(\mathbb{R}, \mathbb{R})$ and $\omega(0)=\omega(T)=0$, then

$$
\int_{0}^{T}|\omega(t)|^{p} d t \leq\left(\frac{T}{\pi_{p}}\right)^{p} \int_{0}^{T}\left|\omega^{\prime}(t)\right|^{p} d t
$$

where $1 \leq p<\infty, \pi_{p}=2 \int_{0}^{(p-1) / p} \frac{d s}{\left(1-\frac{s^{p}}{p-1}\right)^{1 / p}}=\frac{2 \pi(p-1)^{1 / p}}{p \sin (\pi / p)}$.

Remark 2.1 When $p=2, \pi_{2}=2 \int_{0}^{(2-1) / 2} \frac{d s}{\left(1-\frac{s^{2}}{2-1}\right)^{1 / 2}}=\frac{2 \pi(2-1)^{1 / 2}}{2 \sin (\pi / 2)}=\pi$.

Lemma $2.3[20]$ If $x \in C^{2}(\mathbb{R}, \mathbb{R})$ with $x(t+T)=x(t)$, then

$$
\left|x^{\prime}(t)\right|_{2}^{2} \leq\left(\frac{T}{2 \pi}\right)^{2}\left|x^{\prime \prime}(t)\right|_{2}^{2}
$$

Lemma 2.4 If $x \in C^{1}(\mathbb{R}, \mathbb{R})$ with $x(t+T)=x(t)$, and $t_{0} \in[0, T]$ such that $\left|x\left(t_{0}\right)\right|<d$, then

$$
\left(\int_{0}^{T}|x(t)|^{p} d t\right)^{\frac{1}{p}} \leq\left(\frac{T}{\pi_{p}}\right)\left(\int_{0}^{T}\left|x^{\prime}(t)\right|^{p} d t\right)^{\frac{1}{p}}+d T^{\frac{1}{p}} .
$$

Proof Let $\omega(t)=x\left(t+t_{0}\right)-x\left(t_{0}\right)$, and then $\omega(0)=\omega(T)=0$. By Lemma 2.2 and Minkowski's inequality, we have

$$
\begin{aligned}
\left(\int_{0}^{T}|x(t)|^{p} d t\right)^{\frac{1}{p}} & =\left(\int_{0}^{T}\left|\omega(t)+x\left(t_{0}\right)\right|^{p} d t\right)^{\frac{1}{p}} \\
& \leq\left(\int_{0}^{T}|\omega(t)|^{p} d t\right)^{\frac{1}{p}}+\left(\int_{0}^{T}\left|x\left(t_{0}\right)\right|^{p} d t\right)^{\frac{1}{p}} \\
& \leq\left(\frac{T}{\pi_{p}}\right)\left(\int_{0}^{T}\left|\omega^{\prime}(t)\right|^{p} d t\right)^{\frac{1}{p}}+d T^{\frac{1}{p}} \\
& =\left(\frac{T}{\pi_{p}}\right)\left(\int_{0}^{T}\left|x^{\prime}(t)\right|^{p} d t\right)^{\frac{1}{p}}+d T^{\frac{1}{p}}
\end{aligned}
$$

This completes the proof of Lemma 2.4. 
Lemma 2.5 If $x \in C^{2}(\mathbb{R}, \mathbb{R})$ with $x(t+T)=x(t)$, and $t_{0} \in[0, T]$ such that $\left|x\left(t_{0}\right)\right|<d$, then

$$
\left(\int_{0}^{T}|x(t)|^{2} d t\right)^{\frac{1}{2}} \leq\left(\frac{T^{2}}{2 \pi^{2}}\right)\left(\int_{0}^{T}\left|x^{\prime \prime}(t)\right|^{2} d t\right)^{\frac{1}{2}}+d T^{\frac{1}{2}}
$$

Proof From Lemma 2.3 and Lemma 2.4, we know that, when $p=2$, we have

$$
\begin{aligned}
\left(\int_{0}^{T}|x(t)|^{2} d t\right)^{\frac{1}{2}} & \leq\left(\frac{T}{\pi}\right)\left(\int_{0}^{T}\left|x^{\prime}(t)\right|^{2} d t\right)^{\frac{1}{2}}+d T^{\frac{1}{2}} \\
& \leq\left(\frac{T}{\pi}\right)\left(\frac{T}{2 \pi}\right)\left(\int_{0}^{T}\left|x^{\prime \prime}(t)\right|^{2} d t\right)^{\frac{1}{2}}+d T^{\frac{1}{2}} \\
& =\left(\frac{T^{2}}{2 \pi^{2}}\right)\left(\int_{0}^{T}\left|x^{\prime \prime}(t)\right|^{2} d t\right)^{\frac{1}{2}}+d T^{\frac{1}{2}}
\end{aligned}
$$

This completes the proof of Lemma 2.5.

\section{Main results}

First we consider $(1.1)$ when $g(t, x)$ is of attractive type. Assume that

$$
\varphi(t)=\lim _{x \rightarrow+\infty} \sup \frac{g(t, x)}{x}
$$

exists uniformly a.e. $t \in[0, T]$, i.e., for any $\varepsilon>0$ there is $g_{\varepsilon} \in L^{2}(0, T)$ such that

$$
g(t, x) \leq(\varphi(t)+\varepsilon) x+g_{\varepsilon}(t)
$$

for all $x>0$ and a.e. $t \in[0, T]$. Moreover, $\varphi \in C(\mathbb{R}, \mathbb{R})$ and $\varphi(t+T)=\varphi(t)$.

For the sake of convenience, we list the following assumptions which will be used repeatedly in the sequel:

$\left(\mathrm{H}_{1}\right)$ There exist two positive constants $D_{1}<D_{2}$ such that

$$
g(t, x)<0, \quad \text { for all } 0<x<D_{1} ; \quad \text { and } \quad g(t, x)>0, \quad \text { for all } x>D_{2}
$$

$\left(\mathrm{H}_{2}\right)$ (Decomposition condition) $g(t, x)=g_{0}(x)+g_{1}(t, x)$, where $g_{0} \in C((0, \infty) ; \mathbb{R})$ and $g_{1}$ : $[0, T] \times[0, \infty) \rightarrow \mathbb{R}$ is an $L^{2}$-Carathéodory function, i.e., it is measurable in the first variable and continuous in the second variable, and for any $b>0$ there is $h_{b} \in$ $L^{2}\left(0, T ; \mathbb{R}_{+}\right)$such that

$$
\left|g_{1}(t, x)\right| \leq h_{b}(t), \quad \text { a.e. } t \in[0, T], \forall 0 \leq x \leq b
$$

$\left(\mathrm{H}_{3}\right)$ (Strong force condition at $\left.x=0\right) \int_{0}^{1} g_{0}(x) d x=-\infty$.

$\left(\mathrm{H}_{4}\right)$ There exists a positive constant $A$ such that $|f(t, u)| \leq A$, for all $(t, u) \in[0, T] \times \mathbb{R}$.

Theorem 3.1 Assume that (3.2), $h(t, x) \leq 0$, and $\left(\mathrm{H}_{1}\right)-\left(\mathrm{H}_{4}\right)$ hold. We have the following condition: 
$\left(\mathrm{H}_{5}\right) A\left(\frac{T}{2 \pi}\right)+\left|\varphi^{+}\right|_{\infty}\left(\frac{T^{3}}{\pi^{2}}\right)<1$.

Then (1.1) has at least one positive T-periodic solution.

Proof Let $X=\left\{x: \mathbb{R} \rightarrow \mathbb{R}\right.$ is $C^{2}$ and satisfies $\left.x(t+T) \equiv x(t)\right\}$, endowed with the $C^{2}$-norm. Let $Y=L^{2}(0, T ; \mathbb{R})$ with the $L^{2}$-norm.

Let $D(L)=\left\{x \in X: x^{\prime \prime \prime}\right.$ is absolutely continuous on $\left.\mathbb{R}\right\}$ and let $L: D(L) \rightarrow Y$ be the operator defined by

$$
(L x)(t)=x^{\prime \prime \prime}(t), \quad t \in \mathbb{R} .
$$

Define a nonlinear mapping $N: Y \rightarrow Y$ by

$$
(N x)(t)=f\left(t, x^{\prime}\right) x^{\prime \prime}(t)+h(t, x) x^{\prime}(t)-g(t, x(t)) .
$$

Then (1.1) can be converted to the abstract equation $L x+N x=0$. Define the projectors $P: X \rightarrow X$ and $Q: Y \rightarrow Y$ by

$$
P x=\frac{1}{T} \int_{0}^{T} x(s) d s ; \quad Q y=\frac{1}{T} \int_{0}^{T} y(s) d s .
$$

The real number $P x$ and $Q y$ are seen as elements of $X$ and $Y$ inasmuch constant function. It is easy to see that $\operatorname{ker} L=\mathbb{R}, \operatorname{Im} L=\left\{y \in Y: \int_{0}^{T} y(t) d t=0\right\}, \operatorname{ker} Q=\operatorname{Im} L, \operatorname{Im} P=\operatorname{ker} L$, and then $L$ is a Fredholm linear mapping with zero index.

Let $K$ denote the inverse of $\left.L\right|_{\operatorname{ker} P \cap D(L)}$. Then we have

$$
\begin{aligned}
{[K y](t)=} & \frac{t}{2} \int_{0}^{T}(T-s) y(s) d s-\frac{t}{2 T} \int_{0}^{T}(T-s)^{2} y(s) d s-\frac{t^{2}}{2 T} \int_{0}^{T}(T-s) y(s) d s \\
& +\frac{1}{2} \int_{0}^{t}(t-s)^{2} y(s) d s .
\end{aligned}
$$

From (3.3), (3.4), and (3.5), it follows that $Q N$ and $K(I-Q) N$ are continuous, and $Q N(\bar{\Omega})$ is bounded and then $K(I-Q) N(\bar{\Omega})$ is compact for any open bounded $\Omega \subset X$, which means $N$ is $L$-compact on $\bar{\Omega}$.

Now we consider the following (homotopy) family of (1.1):

$$
x^{\prime \prime \prime}+\lambda f\left(t, x^{\prime}\right) x^{\prime \prime}+\lambda h(t, x) x^{\prime}=\lambda g(t, x), \quad \lambda \in[0,1]
$$

i.e., the abstract equation $L x+\lambda N x=0$. We need to show that the set of all possible solutions of the family of (3.6) is, a priori, bounded in $C^{2}(\mathbb{R}, \mathbb{R})$ by a constant independent of $\lambda \in[0,1]$.

Suppose that $x$ is a solution to (3.6) for some $\lambda \in[0,1]$. Let $t^{*}, t_{*}$ be, respectively, the global maximum point and global minimum point of $x^{\prime}(t)$ on [0,T]; Firstly, we consider $x^{\prime}\left(t^{*}\right)=\max _{t \in \mathbb{R}} x^{\prime}(t)=\max _{t \in[0, T]} x^{\prime}(t)$. Since $\int_{0}^{T} x^{\prime}(t) d t=0$, we know that there exist two points $t_{1}, t_{2}$ such that $x^{\prime}\left(t_{1}\right)<0, x^{\prime}\left(t_{2}\right)>0$. So, we get $x^{\prime}\left(t^{*}\right)>x^{\prime}\left(t_{2}\right)>0$. Because $x^{\prime}\left(t^{*}\right)$ is the maximum value of $x^{\prime}(t), x^{\prime \prime}\left(t^{*}\right)=0$. Furthermore, we conclude

$$
x^{\prime \prime \prime}\left(t^{*}\right) \leq 0 .
$$


So, we have

$$
x^{\prime \prime \prime}\left(t^{*}\right)+\lambda h\left(t^{*}, x\left(t^{*}\right)\right) x^{\prime}\left(t^{*}\right)=\lambda g\left(t^{*}, x\left(t^{*}\right)\right),
$$

since $x^{\prime \prime \prime}\left(t^{*}\right) \leq 0$ and $h\left(t^{*}, x\left(t^{*}\right)\right) x^{\prime}\left(t^{*}\right) \leq 0$, and we get

$$
g\left(t^{*}, x\left(t^{*}\right)\right) \leq 0 \text {. }
$$

From $\left(\mathrm{H}_{1}\right)$ we obtain

$$
x\left(t^{*}\right) \leq D_{2}
$$

Similarly, we get

$$
g\left(t_{*}, x\left(t_{*}\right)\right) \geq 0
$$

From $\left(\mathrm{H}_{1}\right)$ we obtain

$$
x\left(t_{*}\right) \geq D_{1} .
$$

From (3.8) and (3.9), we know that there exists a point $\xi \in[0, T]$ such that

$$
D_{1} \leq x(\xi) \leq D_{2}
$$

Therefore, we have

$$
|x(t)|=\left|x(\xi)+\int_{\xi}^{t} x^{\prime}(s) d s\right| \leq D_{2}+\int_{0}^{T}\left|x^{\prime}(s)\right| d s .
$$

Multiplying by $x^{\prime}(t)$ on both sides of (3.6) and integrating from 0 to $T$, we have

$$
\begin{aligned}
& \int_{0}^{T} x^{\prime \prime \prime}(t) x^{\prime}(t) d t+\lambda \int_{0}^{T} f\left(t, x^{\prime}\right) x^{\prime \prime}(t) x^{\prime}(t) d t+\lambda \int_{0}^{T} h(t, x)\left(x^{\prime}(t)\right)^{2} d t \\
& \quad=\lambda \int_{0}^{T} g(t, x(t)) x^{\prime}(t) d t .
\end{aligned}
$$

Since $\int_{0}^{T} x^{\prime \prime \prime}(t) x^{\prime}(t)=-\int_{0}^{T}\left|x^{\prime \prime}(t)\right|^{2} d t$, from $\left(\mathrm{H}_{4}\right)$ and $h(t, x) \leq 0$, we have

$$
\begin{aligned}
\int_{0}^{T}\left|x^{\prime \prime}(t)\right|^{2} d t= & \lambda \int_{0}^{T} f\left(t, x^{\prime}\right) x^{\prime \prime}(t) x^{\prime}(t) d t+\lambda \int_{0}^{T} h(t, x)\left|x^{\prime}(t)\right|^{2} d t \\
& -\lambda \int_{0}^{T} g(t, x(t)) x^{\prime}(t) d t \\
\leq & \lambda \int_{0}^{T} f\left(t, x^{\prime}\right) x^{\prime \prime}(t) x^{\prime}(t) d t-\lambda \int_{0}^{T} g(t, x(t)) x^{\prime}(t) d t \\
\leq & \int_{0}^{T}\left|f\left(t, x^{\prime}\right)\right|\left|x^{\prime \prime}(t)\right|\left|x^{\prime}(t)\right| d t+\int_{0}^{T}|g(t, x(t))|\left|x^{\prime}(t)\right| d t
\end{aligned}
$$


Chang Advances in Difference Equations 2014, 2014:162

Page 7 of 12

$$
\begin{aligned}
& \leq A \int_{0}^{T}\left|x^{\prime \prime}(t)\right|\left|x^{\prime}(t)\right| d t+\int_{0}^{T}|g(t, x(t))|\left|x^{\prime}(t)\right| d t \\
& \leq A \int_{0}^{T}\left|x^{\prime \prime}(t)\right|\left|x^{\prime}(t)\right| d t+\left|x^{\prime}\right|_{\infty} \int_{0}^{T}|g(t, x(t))| d t .
\end{aligned}
$$

For any $\varepsilon>0$, let $g_{\varepsilon} \in L^{2}(0, T)$ be as in (3.2). Thus we have

$$
g^{+}(t, x) \leq\left(\varphi^{+}(t)+\varepsilon\right) x(t)+g_{\varepsilon}^{+}(t)
$$

Therefore,

$$
\int_{0}^{T}\left|g^{+}(t, x)\right| d t \leq\left(\left|\varphi^{+}\right|_{\infty}+\varepsilon\right) \int_{0}^{T}|x(t)| d t+\int_{0}^{T}\left|g_{\varepsilon}^{+}(t)\right| d t .
$$

Since $\int_{0}^{T} g(t, x) d t=0$, we can get $\int_{0}^{T}|g(t, x)| d t=2 \int_{0}^{T}\left|g^{+}(t, x)\right| d t$. So, we have

$$
\int_{0}^{T}|g(t, x)| d t \leq 2\left(\left|\varphi^{+}\right|_{\infty}+\varepsilon\right) \int_{0}^{T}|x(t)| d t+2 \int_{0}^{T}\left|g_{\varepsilon}^{+}(t)\right| d t .
$$

From $x(0)=x(T)$, we know that there exists a point $\xi_{1} \in[0, T]$ such that $x^{\prime}\left(\xi_{1}\right)=0$. So, we have

$$
\left|x^{\prime}\right|_{\infty} \leq \int_{0}^{T}\left|x^{\prime \prime}(t)\right| d t
$$

From (3.12) and the Hölder inequality, we have

$$
\begin{aligned}
\int_{0}^{T}\left|x^{\prime \prime}(t)\right|^{2} d t \leq & A \int_{0}^{T}\left|x^{\prime \prime}(t)\right|\left|x^{\prime}(t)\right| d t+2\left(\left|\varphi^{+}\right|_{\infty}+\varepsilon\right) \int_{0}^{T}|x(t)| d t \int_{0}^{T}\left|x^{\prime \prime}(t)\right| d t \\
& +2 \int_{0}^{T}\left|g_{\varepsilon}^{+}(t)\right| d t \int_{0}^{T}\left|x^{\prime \prime}(t)\right| d t \\
\leq & A\left(\int_{0}^{T}\left|x^{\prime \prime}(t)\right|^{2} d t\right)^{\frac{1}{2}}\left(\int_{0}^{T}\left|x^{\prime}(t)\right|^{2} d t\right)^{\frac{1}{2}} \\
& +2\left(\left|\varphi^{+}\right|_{\infty}+\varepsilon\right) T\left(\int_{0}^{T}|x(t)|^{2} d t\right)^{\frac{1}{2}}\left(\int_{0}^{T}\left|x^{\prime \prime}(t)\right|^{2} d t\right)^{\frac{1}{2}} \\
& +2 T\left(\int_{0}^{T}\left|x^{\prime \prime}(t)\right|^{2} d t\right)^{\frac{1}{2}}\left(\int_{0}^{T}\left|g_{\varepsilon}^{+}(t)\right|^{2} d t\right)^{\frac{1}{2}} .
\end{aligned}
$$

From (3.10) and Lemma 2.5, we have

$$
\left(\int_{0}^{T}|x(t)|^{2} d t\right)^{\frac{1}{2}} \leq\left(\frac{T^{2}}{2 \pi^{2}}\right)\left(\int_{0}^{T}\left|x^{\prime \prime}(t)\right|^{2} d t\right)^{\frac{1}{2}}+D_{2} \sqrt{T} .
$$

From (3.13) and Lemma 2.3, we have

$$
\begin{aligned}
& \int_{0}^{T}\left|x^{\prime \prime}(t)\right|^{2} d t \\
& \quad \leq A\left(\int_{0}^{T}\left|x^{\prime \prime}(t)\right|^{2} d t\right)^{\frac{1}{2}} \cdot\left(\frac{T}{2 \pi}\right)\left(\int_{0}^{T}\left|x^{\prime \prime}(t)\right|^{2} d t\right)^{\frac{1}{2}}
\end{aligned}
$$




$$
\begin{aligned}
& +2 T\left(\left|\varphi^{+}\right|_{\infty}+\varepsilon\right)\left[\left(\frac{T^{2}}{2 \pi^{2}}\right)\left(\int_{0}^{T}\left|x^{\prime \prime}(t)\right|^{2} d t\right)^{\frac{1}{2}}+D_{2} \sqrt{T}\right]\left(\int_{0}^{T}\left|x^{\prime \prime}(t)\right|^{2} d t\right)^{\frac{1}{2}} \\
& +2 T\left(\int_{0}^{T}\left|x^{\prime \prime}(t)\right|^{2} d t\right)^{\frac{1}{2}}\left(\int_{0}^{T}\left|g_{\varepsilon}^{+}(t)\right|^{2} d t\right)^{\frac{1}{2}} \\
= & A\left(\frac{T}{2 \pi}\right) \int_{0}^{T}\left|x^{\prime \prime}(t)\right|^{2} d t+\left(\left|\varphi^{+}\right|_{\infty}+\varepsilon\right)\left(\frac{T^{3}}{\pi^{2}}\right) \int_{0}^{T}\left|x^{\prime \prime}(t)\right|^{2} d t \\
& +\left[2 T\left(\left|\varphi^{+}\right|_{\infty}+\varepsilon\right) D_{2} \sqrt{T}+2 T\left|g_{\varepsilon}^{+}\right|_{2}\right]\left(\int_{0}^{T}\left|x^{\prime \prime}(t)\right|^{2} d t\right)^{\frac{1}{2}} \\
= & {\left[A\left(\frac{T}{2 \pi}\right)+\left(\left|\varphi^{+}\right|_{\infty}+\varepsilon\right)\left(\frac{T^{3}}{\pi^{2}}\right)\right] \int_{0}^{T}\left|x^{\prime \prime}(t)\right|^{2} d t } \\
& +2 T\left[\left(\left|\varphi^{+}\right|_{\infty}+\varepsilon\right) D_{2} \sqrt{T}+\left|g_{\varepsilon}^{+}\right|_{2}\right]\left(\int_{0}^{T}\left|x^{\prime \prime}(t)\right|^{2} d t\right)^{\frac{1}{2}} .
\end{aligned}
$$

Since $\varepsilon$ sufficiently small, from $\left(\mathrm{H}_{5}\right)$ we know that $A\left(\frac{T}{2 \pi}\right)+\left|\varphi^{+}\right|_{\infty}\left(\frac{T^{3}}{\pi^{2}}\right)<1$. Thus, it is easy to see that there exists a positive constant $M_{1}^{\prime}$ such that

$$
\int_{0}^{T}\left|x^{\prime \prime}(t)\right|^{2} d t \leq M_{1}^{\prime}
$$

So, by the Hölder inequality, we have

$$
\left|x^{\prime}\right|_{\infty} \leq \int_{0}^{T}\left|x^{\prime \prime}(t)\right| d t \leq \sqrt{T}\left(\int_{0}^{T}\left|x^{\prime \prime}(t)\right|^{2}\right)^{\frac{1}{2}} \leq \sqrt{T} M_{1}^{\prime \frac{1}{2}}:=M_{2} .
$$

From (3.11), we have

$$
|x|_{\infty} \leq D_{2}+\int_{0}^{T}\left|x^{\prime}(t)\right| d s \leq D_{2}+M_{2} T:=M_{1} .
$$

On the other hand, from $x^{\prime}(0)=x^{\prime}(T)$, we know that there exists a point $\xi_{2} \in[0, T]$ such that $x^{\prime \prime}\left(\xi_{2}\right)=0$. From (3.2), (3.14), and (3.15), and by (3.6), we have

$$
\begin{aligned}
\left|x^{\prime \prime}\right|_{\infty} & \leq \max _{t \in \mathbb{R}}\left|\int_{\xi_{2}}^{t} x^{\prime \prime \prime}(s) d s\right| \\
& \leq \lambda \int_{0}^{T}\left|f\left(t, x^{\prime}\right)\right|\left|x^{\prime \prime}(t)\right| d t+\lambda \int_{0}^{T}|h(t, x)|\left|x^{\prime}(t)\right| d t+\lambda \int_{0}^{T}|g(t, x)| d t \\
& \leq \lambda\left(|f|_{M_{2}} \sqrt{T} M_{1}^{\prime \frac{1}{2}}+|h|_{M_{1}} T M_{2}+2\left(\left|\varphi^{+}\right|_{\infty}+\varepsilon\right) T M_{1}+2 \sqrt{T}\left|g_{\varepsilon}^{+}\right|_{2}\right) \\
& :=\lambda M_{3},
\end{aligned}
$$

where $|h|_{M_{1}}=\max _{|x| \leq M_{1}}|h(t, x)|,|f|_{M_{2}}=\max _{|u| \leq M_{2}}|f(t, u)|$.

Next, multiplying (3.6) by $x^{\prime}(t)$ we get

$$
\begin{aligned}
& x^{\prime \prime \prime}(t) x^{\prime}(t)+\lambda f\left(t, x^{\prime}\right) x^{\prime \prime}(t) x^{\prime}(t)+\lambda h(t, x)\left(x^{\prime}(t)\right)^{2} \\
& \quad=\lambda\left(g_{0}(x(t))+g_{1}(t, x(t))\right) x^{\prime}(t) .
\end{aligned}
$$


Let $\tau \in[0, T]$, for any $\tau \leq t \leq T$, we integrate (3.17) on $[\tau, t]$ and get

$$
\begin{aligned}
\lambda \int_{x(\tau)}^{x(t)} g_{0}(u) d u= & \lambda \int_{\tau}^{t} g_{0}(x(s)) x^{\prime}(s) d s \\
= & x^{\prime \prime}(t) x^{\prime}(t)-x^{\prime \prime}(\tau) x^{\prime}(\tau)-\int_{\tau}^{t}\left|x^{\prime \prime}(s)\right|^{2} d t+\lambda \int_{\tau}^{t} f\left(s, x^{\prime}\right) x^{\prime \prime}(s) x^{\prime}(s) d s \\
& +\lambda \int_{\tau}^{t} h(s, x)\left(x^{\prime}(t)\right)^{2} d t-\lambda \int_{\tau}^{t} g_{1}(s, x(s)) x^{\prime}(s) d s .
\end{aligned}
$$

By (3.14), (3.15), and (3.16) we have

$$
\begin{aligned}
& x^{\prime \prime}(t) x^{\prime}(t) \leq \lambda M_{3} M_{2}, \\
& \left.\left|\int_{\tau}^{t}\right| x^{\prime \prime}(s)\right|^{2} d s \mid \leq \lambda^{2} M_{3}^{2} T, \\
& \left|\int_{\tau}^{t} f\left(s, x^{\prime}\right) x^{\prime \prime}(s) x^{\prime}(s) d s\right| \leq \lambda|f|_{M_{2}} M_{3} M_{2} T, \\
& \left|\int_{\tau}^{t} h(s, x) x^{\prime}(s) x^{\prime}(s) d s\right| \leq|h|_{M_{1}} M_{2}^{2} T, \\
& \left|\int_{\tau}^{t} g_{1}(s, x(s)) x^{\prime}(s) d s\right| \leq \sqrt{T} M_{2}\left|g_{M_{1}}\right|_{2},
\end{aligned}
$$

where $g_{M_{1}}=\max _{0 \leq x \leq M_{1}}\left|g_{1}(t, x)\right| \in L^{2}(0, T)$ is as in $\left(\mathrm{H}_{2}\right)$.

From these inequalities we can derive form (3.18) that

$$
\left|\int_{x(\tau)}^{x(t)} g_{0}(u) d u\right| \leq M_{4}^{\prime},
$$

for some constant $M_{4}^{\prime}$ which is independent on $\lambda, x$ and $t$. In view of the strong force condition $\left(\mathrm{H}_{3}\right)$, we know that there exists a constant $M_{4}>0$ such that

$$
x(t) \geq M_{4}, \quad \forall t \in[\tau, T] .
$$

The case $t \in[0, \tau]$ can be treated similarly.

From (3.8), (3.14), (3.15), (3.16), and (3.20), we let

$$
\Omega=\left\{x \in X: E_{1}<x(t)<E_{2},\left|x^{\prime}(t)\right|<E_{3} \text { and }\left|x^{\prime \prime}(t)\right|<E_{4}, \forall t \in[0, T]\right\}
$$

where $0<E_{1}<\min \left(M_{4}, D_{1}\right), E_{2}>\max \left(M_{1}, D_{2}\right), E_{3}>M_{2}$ and $E_{4}>M_{3}$. Then the conditions (i) and (ii) of Lemma 2.1 are satisfied. For a constant $x \in \operatorname{ker} L, x>0$, we have

$$
Q N x=\frac{1}{T} \int_{0}^{T} g(t, x) d t .
$$

The degree condition $\left(\mathrm{H}_{1}\right)$ shows that

$$
D_{0}\left(\left.Q N\right|_{\operatorname{ker} L}, \Omega \cap \operatorname{ker} L\right)=1 \text {. }
$$


Thus (iii) of Lemma 2.1 is also verified. Therefore $L x+N x=0$ has at least one solution in $\bar{\Omega}$, which means (1.1) has at least one positive $T$-periodic solution.

Next we consider (1.1) when $g(t, x)$ is of repulsive type.

Theorem 3.2 Assume that (3.2), $h(t, x) \leq 0,\left(\mathrm{H}_{2}\right)$, and $\left(\mathrm{H}_{4}\right),\left(\mathrm{H}_{5}\right)$ are satisfied. We have the following condition:

$\left(\mathrm{H}_{1}^{\prime}\right)$ there exist two positive constants $D_{1}<D_{2}$ such that

$$
g(t, x(t))>0, \quad \text { for all } 0<x(t)<D_{1} ; \quad \text { and } \quad g(t, x(t))<0, \quad \text { for all } x(t)>D_{2}
$$

$\left(\mathrm{H}_{3}^{\prime}\right)$ (Strong force condition at $\left.x=0\right) \int_{0}^{1} g_{0}(x) d x=+\infty$.

Then (1.1) has at least one positive T-periodic solution.

Proof Let $\lambda \in[0,1]$ and consider the following:

$$
x^{\prime \prime \prime}+\lambda f\left(t, x^{\prime}\right) x^{\prime \prime}+\lambda h(t, x) x^{\prime}=\lambda g(t, x) \text {. }
$$

Let $t^{*}, t_{*}$ be, respectively, the global maximum point and global minimum point of $x^{\prime}(t)$ on $[0, T]$. First, we consider $x^{\prime}\left(t_{*}\right)=\min _{t \in \mathbb{R}} x^{\prime}(t)=\min _{t \in[0, T]} x^{\prime}(t)$. Since $\int_{0}^{T} x^{\prime}(t) d t=0$, we know that there exist two points $t_{1}, t_{2}$ such that $x^{\prime}\left(t_{1}\right)<0, x^{\prime}\left(t_{2}\right)>0$. So, we get $x^{\prime}\left(t_{*}\right)<$ $x^{\prime}\left(t_{1}\right)<0$. Because $x^{\prime}\left(t_{*}\right)$ is the minimum value of $x^{\prime}(t), x^{\prime \prime}\left(t_{*}\right)=0$. Furthermore, we can conclude

$$
x^{\prime \prime \prime}\left(t_{*}\right) \geq 0 \text {. }
$$

So, we have

$$
x^{\prime \prime \prime}\left(t_{*}\right)+\lambda h\left(t_{*}, x\left(t_{*}\right)\right) x^{\prime}\left(t_{*}\right)=\lambda g\left(t_{*}, x\left(t_{*}\right)\right),
$$

since $x^{\prime \prime \prime}\left(t_{*}\right) \geq 0$ and $h\left(t_{*}, x\left(t_{*}\right)\right) x^{\prime}\left(t_{*}\right) \geq 0$, we get

$$
g\left(t_{*}, x\left(t_{*}\right)\right) \geq 0
$$

Hence, from $\left(\mathrm{H}_{1}^{\prime}\right)$ we know that there exists a positive constant $D_{2}$ such that

$$
x\left(t_{*}\right) \leq D_{2} .
$$

Similarly, we get

$$
g\left(t^{*}, x\left(t^{*}\right)\right) \leq 0
$$

Hence, from $\left(\mathrm{H}_{1}^{\prime}\right)$ we know that there exists a positive constant $D_{1}$ such that

$$
x\left(t^{*}\right) \geq D_{1}
$$


From (3.24) and (3.25), we know that there exists a point $\zeta \in[0, T]$ such that

$$
D_{1} \leq x(\zeta) \leq D_{2}
$$

Therefore, we have

$$
|x(t)|=\left|x(\zeta)+\int_{\zeta}^{t} x^{\prime}(s) d s\right| \leq D_{2}+\int_{0}^{T}\left|x^{\prime}(s)\right| d s
$$

The rest of the proof is the same as that of Theorem 3.1.

\section{Examples}

Finally, we present some examples to illustrate our result.

Example 4.1 Consider the three-order differential equation with singularity:

$$
\begin{aligned}
& x^{\prime \prime \prime}(t)+\frac{1}{5}\left(2 \sin 2 t \cos x^{\prime}(t)+1\right) x^{\prime \prime}(t)+\frac{1}{10}\left(-x^{2}(t)-3\right) x^{\prime}(t) \\
& =\frac{1}{25}(\sin 2 t+3) x(t)-\frac{1}{x(t)^{\kappa}},
\end{aligned}
$$

where $\kappa \geq 1$.

It is clear that $T=\pi, f\left(t, x^{\prime}\right)=\frac{1}{5}\left(2 \sin 2 t \cos x^{\prime}+1\right), h(t, x)=\frac{1}{10}\left(-x^{2}-3\right)<0, g(t, x)=$ $\frac{1}{25}(\sin 2 t+3) x-\frac{1}{x^{r}}, \varphi(t)=\frac{1}{25}(\sin 2 t+3)$. It is obvious that $\left(\mathrm{H}_{1}\right)-\left(\mathrm{H}_{4}\right)$ hold. Now we consider the assumption $\left(\mathrm{H}_{5}\right)$. Since $A \leq \frac{3}{5},\left|\varphi^{+}\right|_{\infty} \leq \frac{4}{25}$, we have

$$
\begin{aligned}
& A\left(\frac{T}{2 \pi}\right)+\left|\varphi^{+}\right|_{\infty}\left(\frac{T^{3}}{\pi^{2}}\right) \\
& \quad \leq \frac{3}{5} \times \frac{\pi}{2 \pi}+\frac{4}{25} \times\left(\frac{\pi^{3}}{\pi^{2}}\right) \\
& \quad=\frac{3}{5} \times \frac{1}{2}+\frac{4}{25} \times \pi \\
& \quad=\frac{15+8 \pi}{50}<1 .
\end{aligned}
$$

So by Theorem 3.1, we know (4.1) has at least one positive $\pi$-periodic solution.

\section{Competing interests}

The author declares that they have no competing interests.

\section{Author's contributions}

ZBC worked together in the derivation of the mathematical results. The author read and approved the final manuscript.

\section{Acknowledgements}

ZBC would like to thank the referee for invaluable comments and insightful suggestions. This work was supported by NSFC project (No. 11326124, 11271339) 


\section{References}

1. Taliaferro, S: A nonlinear singular boundary value problem. Nonlinear Anal. TMA 3, 897-904 (1979)

2. Agarwal, RP, O'Regan, D: Existence theory for single and multiple solutions to singular positone boundary value problems. J. Differ. Equ. 175, 393-414 (2001)

3. Cheng, ZB, Ren, JL: Periodic and subharmonic solutions for Duffing equation with a singularity. Discrete Contin. Dyn. Syst. 32, 1557-1574 (2012)

4. Chu, JF, Torres, P, Zhang, MR: Periodic solution of second order non-autonomous singular dynamical systems. J. Differ Equ. 239, 196-212 (2007)

5. Fonda, A, Manásevich, R: Subharmonics solutions for some second order differential equations with singularities. SIAM J. Math. Anal. 24, 1294-1311 (1993)

6. Martins, RF: Existence of periodic solutions for second-order differential equations with singularities and the strong force condition. J. Math. Anal. Appl. 317, 1-13 (2006)

7. Li, X, Zhang, ZH: Periodic solutions for damped differential equations with a weak repulsive singularity. Nonlinear Anal. TMA 70, 2395-2399 (2009)

8. del Poin, M, Manásevich, R: Infinitely many T-periodic solutions for a problem arising in nonlinear elasticity. J. Differ. Equ. 103, 260-277 (1993)

9. del Poin, M, Manásevich, R, Murua, A: On the number of $2 \pi$-periodic solutions for $u^{\prime \prime}+g(u)=s(1+h(t))$ using the Poincaré-Birkhoff theorem. J. Differ. Equ. 95, 240-258 (1992)

10. Torres, P: Weak singularities may help periodic solutions to exist. J. Differ. Equ. 232, 277-284 (2007)

11. Sun, YP: Positive solution of singular third-order three-point boundary value problem. J. Math. Anal. Appl. 306 586-603 (2005)

12. Chu, JF, Zhou, ZC: Positive solutions for singular non-linear third-order periodic boundary value problems. Nonlinear Anal. TMA 64, 1528-1542 (2006)

13. Sun, JX, Liu, YS: Multiple positive solutions of singular third-order periodic boundary value problem. Acta Math. Sci. 25, 81-88 (2005)

14. Palamides, AP, Smyrlis, G: Positive solutions to a singular third-order three-point boundary value problem with an indefinitely signed Green's function. Nonlinear Anal. TMA 68, 2014-2118 (2008)

15. Li, YX: Positive periodic solutions for fully third-order ordinary differential equations. Comput. Math. Appl. 59, 3464-3471 (2010)

16. Liu, ZQ, Ume, JS, Kang, SM: Positive solutions of a singular nonlinear third order two-point boundary value problem. J. Math. Anal. Appl. 326, 589-601 (2007)

17. Ren, JL, Cheng, ZB, Chen, YL: Existence results of periodic for third-order nonlinear singular differential equation. Math. Nachr. 286, 1022-1042 (2013)

18. Mawhin, J: Topological degree and boundary value problems for nonlinear differental equations. In: Topological Methods for Ordinary Differential Equations. Lecture Notes in Math., vol. 1537, pp. 74-142. Springer, Berlin (1993)

19. Zhang, MR: Nonuniform nonresonance at the first eigenvalue of the $p$-Laplacian. Nonlinear Anal. TMA 29, 41-51 (1997)

20. Ren, JL, Cheng, ZB: On high-order delay differential equation. Comput. Math. Appl. 57, 324-331 (2009)

10.1186/1687-1847-2014-162

Cite this article as: Cheng: Existence of positive periodic solutions for third-order differential equation with strong singularity. Advances in Difference Equations 2014, 2014:162

\section{Submit your manuscript to a SpringerOpen ${ }^{\circ}$ journal and benefit from:}

- Convenient online submission

Rigorous peer review

- Immediate publication on acceptance

- Open access: articles freely available online

- High visibility within the field

- Retaining the copyright to your article 